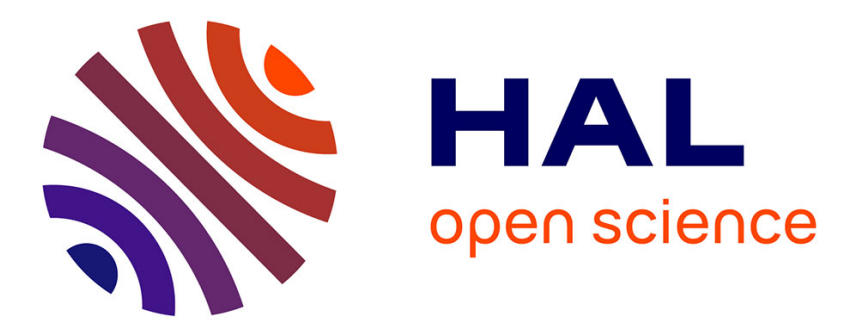

\title{
Diffusion Raman basse fréquence dans les fibres optiques
}

A. Saissy

\section{To cite this version:}

A. Saissy. Diffusion Raman basse fréquence dans les fibres optiques. Revue de Physique Appliquée, 1986, 21 (8), pp.515-521. 10.1051/rphysap:01986002108051500 jpa-00245469

\section{HAL Id: jpa-00245469 https://hal.science/jpa-00245469}

Submitted on 1 Jan 1986

HAL is a multi-disciplinary open access archive for the deposit and dissemination of scientific research documents, whether they are published or not. The documents may come from teaching and research institutions in France or abroad, or from public or private research centers.
L'archive ouverte pluridisciplinaire HAL, est destinée au dépôt et à la diffusion de documents scientifiques de niveau recherche, publiés ou non, émanant des établissements d'enseignement et de recherche français ou étrangers, des laboratoires publics ou privés. 
Classification

Physics Abstracts

$78.30-42.70 \mathrm{C}$

\title{
Diffusion Raman basse fréquence dans les fibres optiques
}

\author{
A. Saissy \\ Laboratoire d'Electrooptique (UA 190), Parc Valrose, 06034 Nice, France
}

(Reçu le 18 octobre 1985, révisé les 30 janvier et 26 mars 1986, accepté le 18 avril 1986)

\begin{abstract}
Résumé. - Nous comparnns la diffusion Raman basse fréquence, 10 à $100 \mathrm{~cm}^{-1}$, de fibres optiques constituées de divers matériaux à structure désordonnée (silice, verre fluoré, polymère). Les propriétés propagatives des fibres pour les ondes lumineuses et acoustiques sont prises en compte dans un modèle proposé par A. J. Martin et W. Brenig. Une longueur de corrélation structurale est déterminée pour chacun des matériaux : $(6,8 \pm 0,3) \AA$ pour $\mathrm{SiO}_{2},(6,4 \pm 0,5) \AA$ pour $\mathrm{ZrF}_{4}-\mathrm{BaF}_{2}$ et $(8,3 \pm 1) \AA$ pour PMMA. L'importance du mode acoustique transverse est confirmée par une étude en lumière polarisée sur fibre biréfringente en silice. L'augmentation de la diffusion avec le dopage dans les fibres à cœur de silice dopée au phosphore est mise en évidence et interprétée qualitativement.
\end{abstract}

\begin{abstract}
We compare the low frequency Raman scattering in silica, fluorozirconate and polymer optical fibres. Fibre properties for optical waves are taken into account in the model proposed by A. J. Martin and W. Brenig. Correlation structural ranges are deduced : $(6.8 \pm 0.3) \AA$ for $\mathrm{SiO}_{2},(6.4 \pm 0.5) \AA$ for $\mathrm{ZrF}_{4}-\mathrm{BaF}_{2}$ and $(8.3 \pm 1) \AA$ for PMMA. The importance of acoustic transverse mode is corroborated by a study in a birefringent silica fibre. Increase of scattering with doping in phosphorus doped silica fibre is observed and qualitatively interpreted.
\end{abstract}

\section{Introduction.}

Les propriétés d'un fibre optique dépendent étroitement des caractéristiques des matériaux qui la composent. En général ces derniers sont des verres dont les pertes optiques résultent de phénomènes d'absorption et de diffusion (Rayleigh, Brillouin et Raman). La structure désordonnée des verres conduit à l'existence d'un type particulier de diffusion dans le domaine spectral $10 \mathrm{~cm}^{-1}$ à $100 \mathrm{~cm}^{-1}$. Cette diffusion a été décrite pour la première fois par A. J. Martin et W. Brenig [1] comme une diffusion de type Brillouin dans un milieu dont les coefficients photoélastiques fluctuent spatialement. L'étude de cette diffusion permet d'accéder à une longueur de corrélation structurale caractéristique du milieu désordonné. Nous possédons ainsi une méthode non destructive permettant d'accéder, in situ, dans le cour d'une fibre optique à un paramètre caractéristique du matériau constituant la fibre et de suivre son évolution lors de la fabrication de la fibre. D'autre part la morphologie d'une fibre affecte la propagation des phonons acoustiques [2] aussi est-il nécessaire de tenir compte des propriétés propagatives de la fibre dans le modèle de A. J. Martin et W. Brenig.
L'objet de la présente note est dans un premier temps une étude comparative de la diffusion Raman basse fréquence sur des fibres constituées de matériaux différents (silice pure, verre fluoré, polymère); par la suite une étude en lumière polarisée est réalisée sur une fibre biréfringente. L'interprétation de ces spectres est conduite à l'aide du modèle Martin-Brenig prenant en compte les propriétés propagatives de la fibre. Nous en déduisons un paramètre d'ordre décrivant les fluctuations spatiales des coefficients photoélastiques que nous comparons aux dimensions des motifs élémentaires utilisés pour décrire la diffusion Raman haute fréquence. Une application au cas des fibres optiques à cour de silice dopée au phosphore est envisagée dans un dernier temps.

\section{Etude expérimentale et résultats.}

Notre étude a été réalisée à l'aide d'un laser à argon accordé sur la longueur d'onde $5145 \AA$ avec une puissance de $100 \mathrm{~mW}$. Le laser est couplé à la fibre par l'intermédiaire d'une lentille de focale $20 \mathrm{~mm}$ pour les fibres multimodes ou d'un objectif de microscope $X 20$ pour la fibre biréfringente. La lumière issue de la face de sortie de la fibre est analysée spectralement 
avec un double monochromateur Jobin-Yvon à réseaux holographiques. L'intensité lumineuse diffusée est détectée par un photomultiplicateur (EMI 95025) suivi d'une chaîne de photocomptage et d'un enregistreur graphique. Par commodité nous avons adopté une configuration de diffusion codirective, néanmoins une étude similaire est aussi possible en configuration de rétrodiffusion.

La figure 1 représente les spectres basse fréquence de trois types de fibre dont les cours sont constitués respectivement de silice pure (FOI code AS133) de verre fluoré (Le Verre Fluoré Sa) et de polyméthacrylate de méthyle (FORETEC). Ce sont des fibres à saut d'indice très multimodes dont les caractéristiques sont données dans le tableau I.

Pour comparer ces spectres nous les avons normalisés sur le maximum de la diffusion sur le domaine spectral $10-100 \mathrm{~cm}^{-1}$; dans ce cas on constate que le minimum de diffusion est le plus faible pour $\mathrm{SiO}_{2}$ mais il est situé à une fréquence plus basse pour le PMMA. Pour le maximum de la diffusion la silice et le verre fluoré se différencient principalement par la position spectrale de ce maximum à 60 et $45 \mathrm{~cm}^{-1}$ respectivement, par contre pour le PMMA on observe deux maxima à 30 et $70 \mathrm{~cm}^{-1}$. Comme il est d'usage

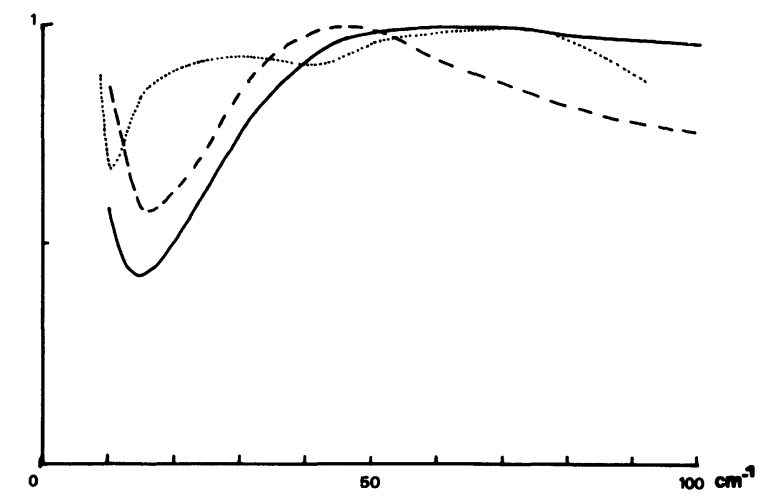

Fig. 1. - Spectres de diffusion Raman basse fréquence de fibres multimodes $-\mathrm{SiO}_{2}----\mathrm{ZrF}_{4} \mathrm{BaF}_{2} \ldots \ldots$ PMMA.

[Low frequency Raman spectra of multimodes fibres $-\mathrm{SiO}_{2}---\mathrm{ZrF}_{4}-\mathrm{BaF}_{2} \ldots \ldots$ PMMA.]

Tableau I. - Diamètres des coeurs des fibres optiques et fréquences principales de diffusion Raman.

[Fibres and Raman scattering parameters.]

\begin{tabular}{|c|c|c|c|}
\hline \multirow{2}{*}{ Fibre } & \multirow{2}{*}{$\begin{array}{l}\text { Diamètre- } \\
\text { cœur } \mu \mathrm{m}\end{array}$} & \multicolumn{2}{|c|}{ Pic principal $\mathrm{cm}^{-1}$} \\
\hline & & fréquence & largeur \\
\hline $\mathrm{SiO}_{2} \mathrm{~F}_{1}$ & 133 & 450 & 200 \\
\hline $\mathrm{ZrF}_{4} \mathrm{BaF}_{2}$ & 65 & 520 & 75 \\
\hline PMMÁ & 250 & 810 & 20 \\
\hline $\mathrm{F}_{2}$ & 60 & & \\
\hline$F_{3}$ & 50 & & \\
\hline
\end{tabular}

pour l'étude des spectres basses fréquences de matériaux amorphes nous interpréterons nos résultats à l'aide des spectres réduits déduits des spectres expérimentaux en divisant par le facteur $w(n(w)+1)$ où $n(w)$ est la distribution de Bose-Enstein.

L'étude en lumière polarisée sur fibre biréfringente, à couur de silice a été entreprise en polarisant successivement la lumière incidente suivant chacun des axes optiques. La face de sortie de la fibre est orientée de manière qu'un axe optique de la fibre soit parallèle à la direction passante d'un polariseur elle-même parallèle à la fente d'entrée du spectromètre. En conservant l'alignement des trois directions - axe optique, polariseur fente d'entrée - pour les deux excitations de la fibre nous nous affranchissons de la variation du facteur de transmission du spectromètre avec la polarisation lumineuse. Cette configuration nous permet donc d'obtenir les spectres polarisé $(X X)$ et dépolarisé $(Y X)$ de la fibre à cour de silice. La bande principale à $450 \mathrm{~cm}^{-1}$ est polarisée [3] le rapport de polarisation de la bande $60 \mathrm{~cm}^{-1}$ est de l'ordre de 0,4. Sur la figure 2 les profils dépolarisé et polarisé de la bande basse fréquence normalisée à l'unité sont comparés, on constate que le rapport 0,4 ci-dessus devient plus faible au voisinage de $100 \mathrm{~cm}^{-1}$.

Une étude similaire a été entreprise sur des fibres multimodes à saut d'indice à cœur de silice dopée au phosphore, figure 3 . Les diamètres de cour des fibres $F_{1}$ et $F_{2}$ sont portés dans le tableau I. En l'absence de mesure absolue de la concentration de phosphore nous avons évalué la valeur relative des concentrations dans les fibres $F_{1}$ et $F_{2}$ en comparant l'intensité des pics de diffusion à $1330 \mathrm{~cm}^{-1}$ et $1140 \mathrm{~cm}^{-1}$, ces pics correspondent à des modes de vibration mettant en jeu l'atome de phosphore (4). Nous avons normalisé nos spectres sur l'intensité de la diffusion, à $450 \mathrm{~cm}^{-1}$, cette bande ne mettant en jeu que les atomes d'oxygène et de silicium de la silice on peut donc supposer qu'elle n'est pas affectée par le dopage au phosphore qui n'est pas très important en technologie des fibres optiques. On constate que la

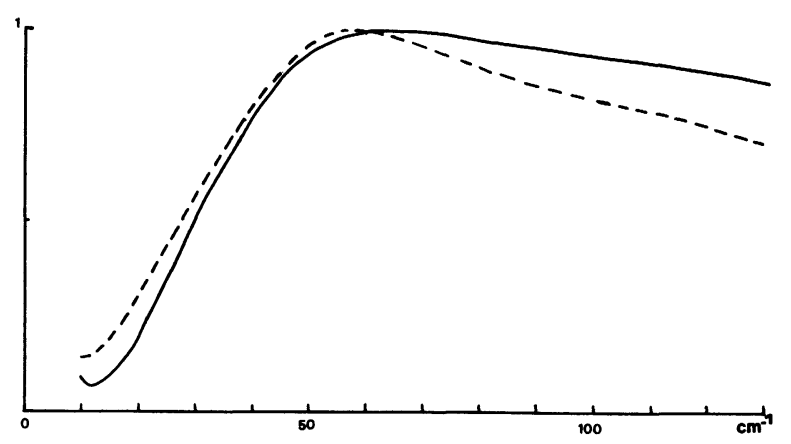

Fig. 2. - Spectres d'une fibre biréfringente en silice polarisé ;---dépolarisé.

[Silica birefringent fibre spectra - polarized ---depolarized.] 


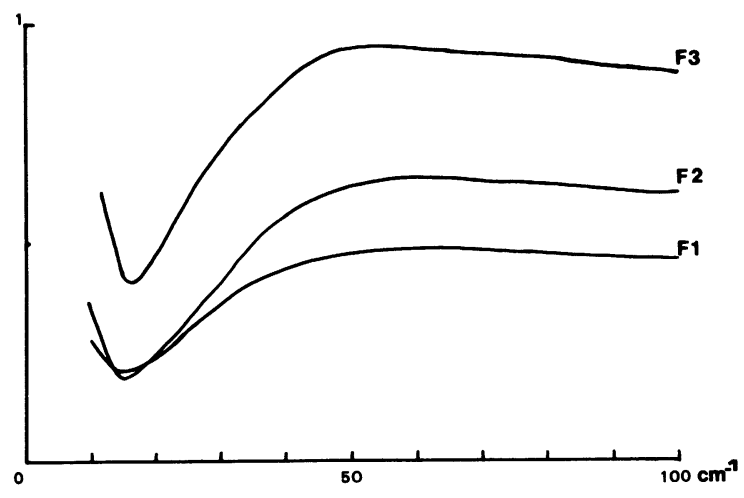

Fig. 3. - Spectres de fibres dopées au phosphore pour trois concentrations $C_{\mathrm{F} 1}<C_{\mathrm{F} 2}<C_{\mathrm{F} 3}$.

[Phosphorus doped silica fibres spectra $C_{\mathrm{F} 1}<C_{\mathrm{F} 2}<C_{\mathrm{F} 3}$.]

diffusion basse fréquence augmente fortement avec le dopage, la position spectrale du maximum étant peu affectée.

\section{Modèle théorique.}

3.1 EXPRESSION DE L'INTENSITÉ DIFFUSÉE. - L'intensité diffusée peut se calculer à l'aide de la théorie des perturbations dans les fibres. Nous supposerons que les modes de la fibre ne sont pas affectés par la diffusion et qu'ils sont linéairement polarisés dans le plan de section droite $(x, y)$. Les ondes lumineuses excitatrice et diffusée s'expriment sous forme de développements modaux.

$$
\mathbf{E}^{(i)}(\mathbf{r}, t)=\sum_{\mu} A^{(i, \mu)} \mathbf{E}_{\mathfrak{t}}^{(i, \mu)}(x, y) \exp j\left(\omega_{i} t-\beta^{(i, \mu)} z\right)
$$

$\beta^{(i, \mu)}$ est la constante de propagation suivant $\mathrm{O} z \mathrm{du}$ mode $\mu$ de profil transversal $\mathbf{E}_{t}^{(i, \mu)}$. Considérons le cas d'un processus Stokes où l'onde excitatrice de pulsation $\omega_{\mathrm{p}}$ interagit avec le milieu matériel pour émettre un phonon de pulsation $\Omega$ et une onde lumineuse de pulsation $\omega_{\mathrm{s}}=\omega_{\mathrm{p}}-\Omega$. Si chaque mode est normalisé de manière à transporter une puissance moyenne unité, la puissance totale diffusée sera

$$
\omega^{(s)}=\sum_{v}\left\langle\left|A^{(s, v}\right|^{2}\right\rangle
$$

où le caractère incohérent de la diffusion est pris en compte par une moyenne d'ensemble soit

$$
\begin{aligned}
&\left\langle\left|A^{(s, \nu)}\right|^{2}\right\rangle=\frac{W_{\mathrm{s}}}{16} \sum_{\mu, \gamma} A^{(p, \mu)} A^{(p, \gamma) *} \times \\
& \times \iiint G^{(p, \mu, \gamma)}(\mathbf{R}, W) \mathrm{d} \mathbf{R} \mathrm{d} W
\end{aligned}
$$

avec

$$
\begin{gathered}
G^{(p, \mu, \gamma}=\iiint_{F^{\prime}} F^{(\mu, v)}(\mathbf{r}, z) F^{*(\gamma, v)}(\mathbf{r}+\mathbf{R}, z+W) \mathrm{d} z \mathrm{~d} \mathbf{r} \\
F^{(\mu, \nu)}=\mathbf{E}_{\mathrm{t}}^{(\mathbf{s}, v) *}(\mathbf{r}) \delta \mathbf{X}^{*}(\mathbf{r}, z) \mathbf{E}_{\mathfrak{t}}^{(p, \mu)}(\mathbf{r}) \exp j\left(\beta^{(s, v)}-\beta^{(p, \mu)}\right) z \\
\mathbf{r}=(x, y, 0), \quad \mathbf{R}=(U, V, 0) .
\end{gathered}
$$

Cette démarche est analogue à celle de P. O'Connor [5], mais ici nous avons conservé la dépendance spatiale de la fluctuation de la susceptibilité contrairement aux auteurs précédents qui se limitent à $\delta X$ constant. L'expression ci-dessus se simplifie si l'on néglige dans le développement de $\left\langle\left|A^{(s, v)}\right|^{2}\right\rangle$ les termes exponentiels à oscillation rapide avec $z$, c'est-à-dire que l'on prendra $\mu=\gamma$ et on ne couplera que des composantes identiques de $\mathbf{E}_{\mathbf{t}}^{(s, v)}$.

3. 2 Diffusion Brillouin. - Pour la diffusion basse énergie qui nous intéresse ici nous adopterons un modèle de diffusion Brillouin. La susceptibilité du milieu est modulée par les phonons acoustiques qui le traversent, cette modulation est liée au tenseur déformation $\mathbf{S}$ par l'intermédiaire du tenseur photoélastique $\mathbf{P}$ soit $\boldsymbol{\delta} \mathbf{X}=\mathbf{P}$.S. Il est commode de séparer $\mathbf{S}$ en une composante de trace nulle notée $\mathbf{J}$ et une composante $\frac{1}{3} \operatorname{Tr} S \mathbf{I}$ proportionnelle au tenseur unité I; dans ce cas

avec

$$
\delta \mathbf{X}(\mathbf{r}, z)=C_{\mathrm{L}} \frac{1}{3} \operatorname{Tr} S \mathbf{I}+C_{\mathrm{T}} \mathbf{J}
$$

$$
C_{\mathrm{L}}=p_{11}+2 p_{12}, C_{\mathrm{T}}=p_{11}-p_{12}
$$

$p_{11}, p_{12}$ étant deux coefficients photoélastiques.

L'existence d'un désordre dans le milieu se traduit par une fluctuation spatiale des coefficients $C_{\mathrm{L}}$ et $C_{\mathrm{T}}$ autour d'une valeur moyenne; la partie de $\delta X$ liée au désordre s'écrira donc

$$
\delta \mathbf{X}(\mathbf{r}, z)=\delta C_{\mathbf{L}}(\mathbf{r}, z) \frac{1}{3} \operatorname{Tr} S \mathbf{I}+\delta C_{\mathbf{T}}(\mathbf{r}, z) \mathbf{J}
$$

où $\delta C_{\mathrm{L}}\left(\right.$ respt. $\left.\delta C_{\mathrm{T}}\right)$ représente la fluctuation de $C_{\mathrm{L}}$ (respt. $C_{\mathrm{T}}$ ). Nous représenterons les variations spatiales de $\delta C$ par une loi gaussienne

$$
\begin{aligned}
& \delta C(\mathbf{r}+\mathbf{R}, z+W) \delta C^{*}(\mathbf{r}, z)= \\
& \quad=|\delta C(\mathbf{r}, z)|^{2} \exp -\frac{R^{2}+W^{2}}{4 \sigma^{2}}
\end{aligned}
$$

$2 \sigma$ étant une longueur de corrélation structurale.

Pour une onde acoustique se propageant suivant la direction $O z$ avec un vecteur d'onde $q_{11}$ l'intensité diffusée relative au mode $\mu$ a pour expression

$$
\begin{aligned}
W_{\mathrm{mn}}^{(v)}= & \frac{\omega_{\mathrm{s}}^{2}}{16}\left|A^{(p)}\right|^{2} \pi(2 \sigma)^{3}\left\{T_{\mathrm{mn}} \delta_{\mathrm{mn}} \overline{\left|\delta C_{\mathrm{L}}\right|^{2}}+\right. \\
& \left.+R_{\mathrm{mn}} \overline{\left|\delta C_{\mathrm{T}}\right|^{2}}\right\} \exp -\sigma^{2}\left(\beta_{\mathrm{m}}^{(\mathrm{s})}-\beta_{\mathrm{n}}^{(\mathrm{p})}+\mathrm{q}_{\|}\right)^{2}
\end{aligned}
$$

où nous avons supposé que $|\mathbf{R}|$ est petit et voisin de $2 \sigma ;$ d'autre part la moyenne $\int_{0}^{\mathrm{L}}|\delta C|^{2} \mathrm{~d} z=L \overline{|\delta C|^{2}}$ est indépendante de $\mathbf{r}$.

$\left[\begin{array}{c}T_{\mathrm{mn}} \\ R_{\mathrm{mn}}\end{array}\right]=\iint\left|E^{(\mathrm{s})}\right|^{2}\left|E^{(\mathrm{p})}\right|^{2}\left|\begin{array}{c}\frac{1}{q}|\operatorname{Tr} S|^{2} \\ \left|J_{\mathrm{mn}}\right|^{2}\end{array}\right| r \mathrm{~d} r \mathrm{~d} \varphi$ 
Les intégrales $T_{\mathrm{mn}}, R_{\mathrm{mn}}$ sont des intégrales de recouvrement que l'on calcule à partir du tenseur déformation $S$.

Martin et Brenig [1] considèrent deux types de fluctuations, celles des constantes photoélastiques et celles des constantes élastiques, avec une même longueur de corrélation $2 \sigma$. La prise en compte d'un deuxième type de fluctuation conduit à ajouter un terme correctif, indépendant de $\sigma$ et $q_{11}$, à chacun des coefficients $|\delta C|^{2}$, l'expression ci-dessus de $W_{\mathrm{mn}}$ reste valable mais avec des coefficients $|\delta C|^{2}$ corrigés.

Le vecteur déplacement $\mathbf{U}(r, \varphi, z)$ de l'onde acoustique se propageant dans la fibre a pour expression [2] en coordonnées cylindriques :

$$
\begin{aligned}
\operatorname{Cas}(a): q_{1}^{2}=\left(\frac{\Omega}{V_{1}}\right)^{2}-q_{\|}^{2}>0 \\
U_{r}=\left\{q_{1} J_{\mathrm{p}}^{\prime}\left(q_{1} r\right) A_{\mathrm{L}}+i q_{\|} J_{\mathrm{p}}^{\prime}\left(q_{\mathrm{s}} r\right) A_{\mathrm{s}}+\frac{p}{r} J_{\mathrm{p}}\left(q_{\mathrm{s}} r\right) A_{\mathrm{t}}\right\}\left(\begin{array}{c}
\cos p \varphi \\
\sin p \varphi
\end{array}\right) \exp -j q_{\|} z \\
\left.U_{\varphi}=\left\{\frac{p}{r} J_{\mathrm{p}}\left(q_{1} r\right) A_{\mathrm{L}}+i \frac{q p}{q_{\mathrm{s}} r} J_{\mathrm{p}}\left(q_{\mathrm{s}} r\right) A_{\mathrm{s}}+q_{\mathrm{s}} J_{\mathrm{p}}^{\prime} q_{\mathrm{s}} r\right) A_{\mathrm{t}}\right\}\left(\begin{array}{c}
-\sin p \varphi \\
\cos p \varphi
\end{array}\right) \exp -j q_{\|} z \\
U_{z}=\left\{-i q_{\|} J_{\mathrm{p}}\left(q_{1} r\right) A_{\mathrm{L}}-q_{\mathrm{s}} J_{\mathrm{p}}\left(q_{\mathrm{s}} r\right) A_{\mathrm{s}}\right\}\left(\begin{array}{c}
\cos p \varphi \\
\sin p \varphi
\end{array}\right) \exp -j q_{\|} z \\
\operatorname{Cas}(b): q_{\mathrm{l}}^{2}=q_{\|}^{2}-\left(\frac{\Omega}{V_{\mathrm{L}}}\right)^{2}>0 \\
U_{r}=\left\{\left\{q_{1} I_{\mathrm{p}}^{\prime}\left(q_{1} r\right) A_{\mathrm{L}}+i q_{\|} J_{\mathrm{p}}^{\prime}\left(q_{\mathrm{s}} r\right) A_{\mathrm{s}}+\frac{p}{r} J_{\mathrm{p}}\left(q_{\mathrm{s}} r\right) A_{\mathrm{t}}\right\}\left(\begin{array}{c}
\cos p \varphi \\
\sin p \varphi
\end{array}\right) \exp -j q_{\|} z\right. \\
U_{\varphi}=\left\{\frac{p}{r} I_{\mathrm{p}}\left(q_{1} r\right) A_{\mathrm{L}}+i \frac{q p}{q_{\mathrm{s}} r} J_{\mathrm{p}}\left(q_{\mathrm{s}} r\right) A_{\mathrm{s}}+q_{\mathrm{s}} J_{\mathrm{p}}^{\prime}\left(q_{\mathrm{s}} r\right) A_{\mathrm{t}}\right\}\left(\begin{array}{c}
-\sin p \varphi \\
\cos p \varphi
\end{array}\right) \exp -j q_{\|} z \\
U_{z}=\left\{-i q_{\|} I_{\mathrm{p}}\left(q_{1} r\right) A_{\mathrm{L}}-q_{\mathrm{s}} J_{\mathrm{p}}\left(q_{\mathrm{s}} r\right) A_{\mathrm{s}}\right\}\left(\begin{array}{c}
\cos p \varphi \\
\sin p \varphi
\end{array}\right) \exp -j q_{\|} z
\end{aligned}
$$

où $q_{\mathrm{s}}^{2}=\left(\frac{\Omega}{V_{\mathrm{T}}}\right)^{2}-q_{\|}^{2}, q^{2}=q_{\mathrm{s}}^{2}+q_{\|}^{2}, p$ est un entier, $J_{\mathrm{p}}$ et $I_{\mathrm{p}}$ des fonctions de Bessel de $1^{\text {re }}$ espèce normale et modifiée, $V_{\mathrm{L}}$ et $V_{\mathrm{T}}$ les vitesses du son longitudinale et transverse dans le coeur de la fibre. L'expression du tenseur déformation est donnée dans l'appendice A1. Les coefficients $A_{\mathrm{L}}, A_{\mathrm{s}}$ et $A_{\mathrm{t}}$ peuvent s'exprimer en appliquant les conditions de continuité à l'interface coeur-gaine, dans un but de simplification nous les exprimerons en fonction de la puissance acoustique dans le cœur de la fibre soit

$$
W_{\text {coeur }}^{(\mathrm{a})}=\iint R_{3} r \mathrm{~d} r \mathrm{~d} \varphi
$$

où le vecteur de Poynting acoustique a pour expression

$$
R_{3}=\frac{1}{2} \Omega \text { Ré }\left\{j\left(T_{3 r} U_{r}^{*}+T_{3 \varphi} U_{\varphi}^{*}+T_{33} U_{3}^{*}\right)\right\}
$$

$T$ étant le tenseur des contraintes.

Cette puissance peut aussi s'exprimer en fonction du nombre de phonons traversant le coeur de la fibre avec un vecteur d'onde compris entre $q_{11}$ et $q_{11}+\mathrm{d} q_{11}$ soit

$$
\mathrm{d} W_{\text {coeur }}^{\text {(a) }}=\hbar \Omega \pi a^{2} \frac{\Omega}{q_{\|}}(n(\Omega)+1) g\left(q_{\|}\right) \mathrm{d} q_{\|}
$$

où $n(\Omega)$ est la distribution de Bose-Enstein des phonons et $g\left(q_{\|}\right) \mathrm{d} q_{\|}$la densité d'état des phonons que l'on peut prendre égale à $(2 \pi)^{-3} q_{\|}^{2} \mathrm{~d} q_{\|}$. Pour le mode acoustique caractérisé par l'entier $p=0$ il n'y a pas de variation azimutale de l'amplitude acoustique, l'onde acoustique se situe au voisinage de l'axe de la fibre. Pour ce mode nous pouvons exprimer les coefficients $\left|A_{\mathrm{L}}\right|^{2}$ et $\left|A_{\mathrm{T}}\right|^{2}$ en fonction du rapport $\alpha=\left|A_{\mathrm{L}}\right|^{2} / W_{\text {coeur }}^{(\mathrm{a})}$ et calculer l'intensité diffusée.

3.3 CAS D'Une fibre multimode. - Ce type de fibre ne conserve pas la polarisation lumineuse aussi est-il nécessaire de faire une moyenne de l'intensité diffusée sur les différents états de polarisation. Nous obtenons une intensité diffusée dans l'intervalle spectral $\mathrm{d} \Omega$ donnée par l'expression

$$
\begin{aligned}
\frac{\partial W}{\partial \Omega}= & \frac{\omega_{\mathrm{s}}^{2}}{16}\left|A^{(p)}\right|^{2} \frac{\hbar(n(\Omega)+1)}{4 \pi \rho} 2 \sigma \Omega^{3} \times \\
& \times \sum_{i=\mathrm{T}, \mathrm{L}} \frac{1}{V_{i}^{5}} \sum_{j=\mathrm{T}, \mathrm{L}} \gamma_{i j} \overline{\left|\delta C_{i j}\right|^{2}} \exp \left[-\left(\frac{\sigma \Omega}{V_{i}}\right)^{2}\right] .
\end{aligned}
$$

Les coefficients $\gamma_{i j}$ sont des fonctions des intégrales de recouvrement entre le mode acoustique et les modes optiques de la fibre, nous les expliciterons dans le cas d'une fibre monomode. 
Il est commode [6] de mettre l'intensité diffusée sous la forme

$$
\frac{\partial W}{\partial \Omega}=I_{0} \frac{n(\Omega)+1}{\Omega} \sum_{b} C_{b}(\Omega) g_{b}(\Omega)
$$

où $C_{b}(\Omega)$ est une constante de couplage qui décrit l'interaction de la lumière avec la matière. Dans le cas ci-dessus nous aurons

$$
\begin{aligned}
& C(\Omega) \simeq(\sigma \Omega)^{2} \sum_{i=\mathrm{T}, \mathrm{L}} \frac{1}{V_{i}^{5}} \sum_{j=\mathrm{T}, \mathrm{L}} \gamma_{i j} \overline{\left|\delta C_{i j}\right|^{2}} \times \\
& \times \exp \left[-\left(\frac{\sigma \Omega}{V_{i}}\right)^{2}\right]
\end{aligned}
$$

que l'on peut comparer aux spectres réduits portés sur la figure 4. Dans la mesure où $V_{\mathrm{T}}<V_{\mathrm{L}}$ la courbe $C(\Omega)$ présente un maximum pour $\sigma \Omega / V_{\mathrm{T}}=1$, relation que l'on peut utiliser pour déterminer $\sigma$. Martin et Brenig out développé leur modèle dans l'hypothèse où $\sigma \Omega / V_{\mathrm{T}}<1$. Néanmoins R. J. Némanich [7] utilise l'égalité $\sigma \Omega / V_{\mathrm{T}}=1$ pour déterminer $\sigma$ dans les chalcogénures amorphes, l'accord des résultats ainsi obtenus avec la diffusion Raman haute fréquence peut servir à justifier a posteriori l'emploi de l'égalité cidessus.

3.4 CAS D'UNe FIBRe BIRÉFrINGENTE. - Pour ce type de fibre il est possible d'entreprendre une étude en lumière polarisée, nous avons donc calculé le rapport de dépolarisation

$$
\rho=\frac{\partial W^{X Y} / \partial \Omega}{\partial W^{X X} / \partial \Omega}=\frac{\sum_{i} \frac{1}{V_{i}^{5}} \overline{\left|\delta C_{i}\right|^{2}} \gamma_{i \mathrm{~T}}^{(X Y)} \mathrm{e}^{-\sigma^{2} \Omega^{2} / V_{i}^{2}}}{\sum_{i=\mathbf{L}, \mathbf{T}} \frac{\mathrm{e}^{-\sigma^{2} \Omega^{2} / V_{l}^{2}}}{V_{i}^{5}} \sum_{j=\mathbf{L}, \mathbf{T}} \gamma_{i j}^{(X X)} \overline{\left|\delta C_{j}\right|^{2}}}
$$

où les coefficients $\gamma$ sont donnés par les expressions ci-dessous :

$$
\begin{aligned}
& \text { Mode transverse } V_{\|}=V_{\mathrm{T}} \\
& \gamma_{\mathrm{TL}}^{(X X)}=\frac{2 \pi \alpha}{q[\mathrm{TL}]} x^{4} U_{0} \\
& \gamma_{\mathrm{TT}}^{(X X)}=\frac{\pi \alpha}{4[\mathrm{TL}]}\left\{\left(1-x^{2}\right)^{2} U_{2}+2\left(1-\frac{1}{3} x^{2}\right)^{2} U_{0}\right\} \\
& \gamma_{\mathrm{TT}}^{(X Y)}=\frac{\pi \alpha}{4[\mathrm{TL}]}\left(1-x^{2}\right)^{2} U_{2}
\end{aligned}
$$

avec

$$
\begin{gathered}
x=\frac{V_{\mathrm{T}}}{V_{\mathrm{L}}} \\
U_{0}=\left\langle I_{0}^{2}\left(q_{1} r\right)\right\rangle=\iint\left|E^{(\mathbf{s})}\right|^{2}\left|E^{(\mathbf{p})}\right|^{2} I_{0}^{2}\left(q_{1} r\right) r \mathrm{~d} r \\
U_{2}=\left\langle I_{2}^{2}\left(q_{1} r\right)\right\rangle .
\end{gathered}
$$

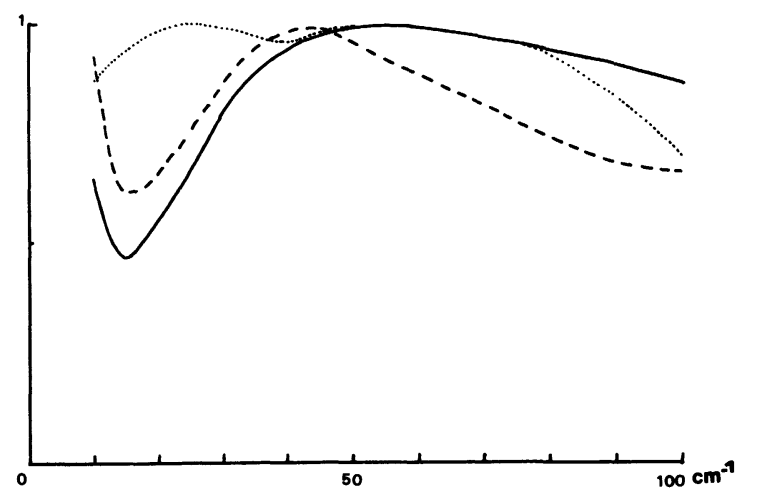

Fig. 4. - Spectres réduits normalisés $-\mathrm{SiO}_{2}---$ $\mathrm{ZrF}_{4}-\mathrm{BaF}_{2} \ldots \ldots$ PMMA.

[Reduced spectra $-\mathrm{SiO}_{2}---\mathrm{ZrF}_{4}-\mathrm{BaF}_{2} \ldots \ldots$ PMMA.]

Mode longitudinal $V_{\|}=V_{\mathrm{L}}$

$$
\begin{aligned}
\gamma_{\mathrm{LT}}^{(X X)}= & \frac{2 \pi \alpha}{q[\mathrm{LL}]} V_{0} \\
\gamma_{\mathrm{LT}}^{(X Y)}= & \frac{\pi(1-\alpha)}{4[\mathrm{LS}]}\left(\frac{x^{2}-1}{x^{2}}\right)^{2} V_{2} \\
\gamma_{\mathrm{LL}}^{(X Y)}= & \frac{2 \pi \alpha}{q[\mathrm{LL}]} V_{0}+\frac{\pi(1-\alpha)}{4[\mathrm{LT}]}\left(\frac{x^{2}-1}{x^{2}}\right)^{2}\left(V_{2}+2 V_{0}\right) \\
& V_{0}=\left\langle J_{0}^{2}\left(q_{\mathrm{s}} r\right)\right\rangle, \quad V_{2}=\left\langle J_{2}^{2}\left(q_{\mathrm{s}} r\right)\right\rangle .
\end{aligned}
$$

Les coefficients $[i j]$ sont donnés dans l'appendice B.

Si l'on se place dans l'hypothèse où $x \ll 1$, cas de la silice, nous obtenons

$$
\rho \approx \frac{\gamma_{\mathrm{TT}}^{(X Y)}}{\gamma_{\mathrm{TT}}^{(X X)}}=1 /\left[1+2\left(1-\frac{1}{3} x^{2}\right)^{2} \frac{U_{0}}{U_{2}}\right] .
$$

Pour un mode acoustique transverse le vecteur d'onde $q_{1}$ est grand donc $U_{0} \sim U_{2}$ il en résulte que le rapport de dépolarisation prend la valeur $1 / 3$ au maximum de la diffusion Raman basse fréquence.

\section{Discussion.}

La contribution d'un mode acoustique est d'autant plus importante que sa vitesse est faible; dans $\mathrm{SiO}_{2}$ et VF. $V_{L}$ est important et sa contribution négligeable : les spectres ne présentent qu'un maximum; pour le PMMA $V_{\mathrm{L}}$ est de l'ordre de grandeur de $V_{\mathrm{T}}$ dans $\mathrm{SiO}_{2}$; le spectre expérimental correspondant présente deux maxima. A partir de la vitesse acoustique et de la position du maximum de diffusion sur le spectre réduit il est possible de déduire la valeur de $2 \sigma$ à l'aide de la relation $2 \pi \rightarrow \omega_{\mathrm{M}} \sigma=V_{\mathrm{T}}$. Dans le tableau II nous constatons que la longueur de corrélation $2 \sigma$ correspond à environ deux fois les dimensions d'un motif élémentaire pour les verres inorganiques; au- 
Tableau II. - Propriétés des matériaux constituant le coeur des fibres.

[Materials properties of fibres.]

\begin{tabular}{|c|c|c|c|c|c|c|c|}
\hline Fibre & $\begin{array}{c}v_{\mathrm{t}} \\
\mathrm{m} / \mathrm{s}\end{array}$ & $\begin{array}{c}v_{1} \\
\mathrm{~m} / \mathrm{s}\end{array}$ & $\begin{array}{r}\Omega \\
\mathrm{cm}\end{array}$ & & & $\sigma$ & $\begin{array}{c}\text { Liaisons } \\
\AA\end{array}$ \\
\hline $\mathrm{SiO}_{2}$ & 3700 & 5900 & 55 & 60 & 7,1 & 6,5 & $\begin{array}{l}\text { Si-O } \sim 1,6 \\
\mathrm{O}-\mathrm{O} \sim 2,61\end{array}$ \\
\hline V.F. & 2250 & 4150 & 35 & 40 & 6,8 & 5,9 & $\begin{array}{c}\mathrm{Ba}-\mathrm{F} \sim 2,8 \\
\mathrm{Zr}-\mathrm{F} \sim 2,1\end{array}$ \\
\hline PMMA & 1570 & 3150 & 18 & 23 & 9,2 & 7,2 & \\
\hline
\end{tabular}

delà le désordre commence à se manifester. Dans le cas du PMMA l'application directe du modèle théorique devrait nous permettre de trouver la position du deuxième maximum de diffusion, $\omega_{\mathrm{M} 2}$, à partir du premier, $\omega_{\mathrm{M} 1}$, avec la relation $\omega_{\mathrm{M} 2}=\frac{V_{\mathrm{L}}}{V_{\mathrm{T}}} \omega_{\mathrm{M} 1}$. Sur le spectre réduit du PMMA nous avons $\omega_{\mathbf{M} 1} \sim$ $25 \mathrm{~cm}^{-1}$; avec la relation $V_{\mathrm{L}} / V_{\mathrm{T}} \sim 2$, nous obtenons $\omega_{\mathrm{M} 2} \sim 50 \mathrm{~cm}^{-1}$ qui est de l'ordre de grandeur de la valeur expérimentale de $60 \mathrm{~cm}^{-1}$. Aux erreurs d'expériences près la théorie est en accord avec l'expérience.

Le rôle important joué par le mode acoustique transverse résulte de son grand vecteur d'onde qui le rend capable d'exciter un grand nombre de défauts lors de la diffusion lumineuse. Il est connu que les modes transverses dépolarisent la lumière diffusée, nous avons pu vérifier cette propriété par une étude en lumière polarisée sur une fibre biréfringente en silice. Nous mesurons au maximum de diffusion $\left(\sim 60 \mathrm{~cm}^{-1}\right)$ un rapport des intensités $W^{(v x)} / W^{(x x)}$ de 0,4 ce qui est voisin de 0,33 calculé théoriquement, l'écart théorie-expérience peut s'expliquer par le fait que la fibre ne conserve pas parfaitement la polarisation lorsqu'elle est excitée suivant un axe. Le modèle théorique et l'expérience s'accordent sur une diminution du rapport ci-dessus pour des fréquences supérieures au maximum de diffusion $\left(\sim 60 \mathrm{~cm}^{-1}\right)$ c'est-à-dire au voisinage du mode acoustique longitudinal. Ainsi le rôle du mode acoustique longitudinal est mis en évidence bien que la fréquence de son maximum de diffusion soit proche des fréquences associées aux bandes Raman hautes fréquences.

Si l'on s'intéresse aux fréquences inférieures à $15 \mathrm{~cm}^{-1}$ le modèle Martin-Brenig est en défaut car il donne une intensité diffusée tendant vers zéro alors qu'on observe un minimum suivi d'une augmentation. Un modèle plus adéquat pour ce domaine spectral est celui des systèmes à deux niveaux $[8,9]$ développé pour interpréter l'excès de diffusion aux très basses fréquences. D'autre part les mesures d'atténuation ultra-sonore à basse température indiquent que la densité spectrale des systèmes à deux niveaux est plus grande dans le verre fluoré que dans la silice [10].
Pour $10 \mathrm{~cm}^{-1}<W<15 \mathrm{~cm}^{-1}$ les spectres réduits de la figure 4 nous indiquent que la fibre en verre fluoré diffuse plus que la fibre en silice pure, ce résultat est donc compatible avec la présence de systèmes à deux niveaux dans le verre fluoré.

L'étude de la diffusion Raman basse fréquence permet donc de caractériser, in situ, la structure des matériaux constituant les fibres, aussi nous avons envisagé une étude en fonction du dopage dans les fibres en silice. Les spectres de la silice dopée au phosphore, figure 3, nous montrent que la fréquence du maximum de diffusion bouge peu, seule son intensité varie. En effet la position du maximum dépend de $2 \sigma$, cette longueur est déjà petite dans la silice pure, il n'est donc guère possible de la diminuer par dopage.

Pour expliquer l'augmentation de la diffusion on peut alors supposer que le nombre de défauts reste fixe, $2 \sigma$ constant, par contre le phosphore pourrait modifier certains défauts de manière à augmenter leur contribution à la diffusion. Par exemple une liaison $\mathrm{Si}-\mathrm{O}-\mathrm{Si}$ brisée qui constitue un défaut pour la silice pure peut être soudée par le phosphore pour donner une liaison Si-O-P, ainsi nous avons remplacé un défaut mécanique par un défaut chimique. Une deuxième situation correspond au cas où un tétraèdre $\mathrm{SiO}_{4}$ est remplacé par. un tétraèdre $\mathrm{PO}_{4}$ possédant une double liaison $\mathrm{P}=\mathrm{O}$ pour satisfaire la valence $5 \mathrm{du}$ phosphore, un désordre électrique est ainsi introduit par le dopage. Nous vérifions bien une augmentation simultanée de l'intensité des bandes $60 \mathrm{~cm}^{-1}$ et $1330 \mathrm{~cm}^{-1}(P=0)$. L'étude de l'évolution de la diffusion Raman basse fréquence dans la silice dopée au phosphore avec la concentration de phosphore est donc compatible avec les renseignements déduits de la partie haute fréquence du spectre.

\section{Conclusion.}

Nous avons développé un modèle de diffusion Raman basse fréquence adapté au cas des fibres optiques. La confrontation de ce modèle avec les résultats expérimentaux obtenus avec quelques matériaux utilisés en technologie des fibres optiques (silice, verre fluoré, polymère...) nous a permis de mesurer une longueur de corrélation caractéristique du désordre présent dans ces matériaux. Dans le cas des verres inorganiques cette longueur correspond à environ deux fois les dimensions des motifs structuraux élémentaires utilisés pour décrire la partie haute fréquence du spectre Raman. Ce modèle rend bien compte de l'évolution du maximum de diffusion avec la vitesse du mode acoustique transverse, le rôle important de ce dernier a été par ailleurs confirmé par une mesure en lumière polarisée.

L'utilisation de la diffusion Raman basse fréquence comme moyen de caractérisation du désordre a été envisagée dans le cas de la silice dopée au phosphore. Nous avons mis en évidence une augmentation de cette diffusion avec la concentration de phosphore, ce résultat a été interprété qualitativement comme une 
augmentation de la section efficace de diffusion des défauts de la silice pure en présence de phosphore.

Cette étude nous a permis de dégager les principes généraux permettant d'interpréter la diffusion Raman basse fréquence dans une fibre optique, l'étude du lien entre cette diffusion et les principaux paramètres régissant la fabrication d'une fibre nous permettra de disposer d'un nouveau moyen de caractérisation, in situ, des fibres optiques.

Appendice A. EXPRESSION DU TENSEUR DÉFORMATION.

$S_{r r}=\frac{\partial U_{r}}{\partial r}, \quad S_{\varphi \varphi}=\frac{U_{r}}{r}+\frac{1}{r} \frac{\partial U_{\varphi}}{\partial \varphi}, \quad S_{z z}=\frac{\partial U_{z}}{\partial z}$

$S_{\varphi r}=\frac{1}{2}\left(\frac{1}{r} \frac{\partial U_{r}}{\partial \varphi}+\frac{\partial U_{\varphi}}{\partial r}-\frac{U_{\varphi}}{r}\right), \quad S_{r z}=\frac{\partial U_{r}}{\partial z}+\frac{\partial U_{z}}{\partial r}$

$S_{\varphi z}=\frac{\partial U_{\varphi}}{\partial z}+\frac{1}{r} \frac{\partial U_{z}}{\partial \varphi}$.

\section{Appendice B.}

EXPRESSIONS APPROCHÉES DES COEFFICIENTS [ii].

$$
\begin{gathered}
x=\frac{V_{\mathrm{T}}}{V_{\mathrm{L}}} \\
-V_{\|} \approx V_{\mathrm{L}}
\end{gathered}
$$

$[\mathrm{LL}]=J_{1}^{2}\left(q_{\mathrm{L}} a\right)+J_{0}^{2}\left(q_{\mathrm{L}} a\right)$

$[\mathrm{LT}]=J_{1}^{2}\left(q_{\mathrm{T}} a\right)+2\left[1-x^{2}\right] J_{0}^{2}\left(q_{\mathrm{T}} a\right)-$ $-\left[2 x^{2}-1\right] J_{2}\left(q_{\mathrm{T}} a\right) J_{0}\left(q_{\mathrm{T}} a\right)$ $-V_{\|} \approx V_{\mathrm{T}}$

$[\mathrm{TT}]=J_{1}^{2}\left(q_{\mathrm{T}} a\right)-J_{2}\left(q_{\mathrm{T}} a\right) J_{0}\left(q_{\mathrm{T}} a\right)$

$[\mathrm{TL}]=-I_{1}^{2}\left(q_{\mathrm{L}} a\right)+\left[3-2 x^{2}\right] I_{0}^{2}\left(q_{\mathrm{L}} a\right)+$ $+2\left[1-x^{2}\right] I_{0}\left(q_{\mathrm{L}} a\right) I_{2}\left(q_{\mathrm{L}} a\right)$.

\section{Bibliographie}

[1] Martin, A. J., Brenig, W., Phys. Status Solidi B 64 (1974) 163.

[2] Thomas, P. J., Rowell, N. L., van Driel, H. M., Stegeman, G. J., Phys. Rev. B 19 (1979) 4986.

[3] Saissy, A., Botineau, J., Ostrowsky, D., J. Physique Lett. 44 (1983.) L-1035-38.

[4] Saissy, A., Botineau, J., Azema, A., Gires, F., J. Physique Lett. 40 (1979) L355-58.

[5] O’Connor, P., Tauc, J., Appl. Opt. 17 (1978) 3226.
[6] Shuker, R., Gammon, R. W., Phys. Rev. Lett. 25 (1970) 222.

[7] Nemanich, R. J., Phys. Rev. 16 (1977) 1655.

[8] Winterling, G., Phys. Rev. B 12 (1975) 2432.

[9] Théodorakopoulos, N., Jackle, J., Phys. Rev. B 14 (1976) 2637.

[10] Doussineau, P., Matecki, M., Schon, W., J. Physique 44 (1983) 101. 